\title{
A COMUNICAÇÃO TERAPÊUTICA COM PACIENTES EM TRANSPLANTE DE MEDULA ÓSSEA: PERFIL DO COMPORTAMENTO VERBAL E EFEITO DE ESTRATÉGIA EDUCATIVA*
}

Tauani Zampieri Fermino', Emilia Campos de Carvalho²

RESUMO: Buscando contribuir na aquisição de competência comunicativa, foram objetivos: verificar a utilização da comunicação terapêutica pela equipe de enfermagem e verificar o efeito de uma estratégia educativa no conhecimento sobre comunicação terapêutica. Inicialmente, com desenho descritivo, foi observado o perfil da comunicação entre 11 profissionais e 20 pacientes, considerando as categorias de comunicação terapêutica e não terapêutica. A de expressão $(80,8 \%)$ foi a mais freqüente, mas ocorreram categorias não terapêuticas (13,6\%). Na etapa seguinte, de desenho quase experimental, observou-se diferença significante em quatro das 22 categorias terapêuticas e em uma das 10 não terapêuticas, ao se comparar o desempenho antes e após a intervenção educativa no padrão de identificação das técnicas de comunicação usadas em simulações em vídeo.

PALAVRAS-CHAVE: Comunicação; Enfermagem; Transplante de Medula Óssea.

\section{THERAPEUTIC COMMUNICATION WITH BONE MARROW TRANSPLANTATION PATIENTS: A PROFILE OF VERBAL BEHAVIOR AND THE EFFECT OF AN EDUCATIONAL STRATEGY}

\begin{abstract}
As an attempt to contribute to the acquisition of communicative competency, this study aimed to: verify the use of therapeutic communication by the nursing team; verify the effect of an educational strategy on knowledge about therapeutic communication. Initially, using a descriptive design, the communication profile among 11 professionals and 20 patients was observed, considering the categories of therapeutic and non-therapeutic communication. Expression was the most frequent category (80.8\%), but non-therapeutic categories occurred (13.6\%). In the following phase, using a quasi-experimental design, a significant difference was observed in four of the 22 therapeutic and in one of the 10 non-therapeutic categories by comparing the performance before and after the educational intervention in the identification pattern of communication techniques used in video simulations.
\end{abstract}

KEYWORDS: Communication; Nursing; Bone Marrow Transplantation.

\section{LA COMUNICACIÓN TERAPÉUTICA CON PACIENTES EN TRASPLANTE DE MÉDULA ÓSEA: PERFIL DEL COMPORTAMIENTO VERBAL Y EFECTO DE ESTRATEGIA EDUCATIVA}

RESUMEN: A fin de contribuir con la adquisición de competencia comunicativa, los objetivos fueron los de verificar la utilización de la comunicación terapéutica por el equipo de enfermería; verificar el efecto de una estrategia educativa en el conocimiento sobre comunicación terapéutica. Inicialmente, con diseño descriptivo, se observó el perfil de la comunicación entre 11 profesionales y 20 pacientes, considerando las categorías de comunicación terapéutica y no terapéutica. La de expresión $(80,8 \%)$ fue la más frecuente, pero ocurrieron categorías no terapéuticas $(13,6 \%)$. En la próxima etapa, de diseño casi experimental, se observó diferencia significante en cuatro de las 22 categorías terapéuticas y en una de las 10 no terapéuticas, al compararse el desempeño antes y después de la intervención educativa, en el patrón de identificación de las técnicas de comunicación usadas en simulaciones en video.

PALABRAS CLAVE: Comunicación, Enfermería, Trasplante de médula ósea.

\footnotetext{
"Pesquisa vinculada ao projeto "Comunicação e enfermagem: as interfaces" da Escola de Enfermagem de Ribeirão Preto - USP (EERP-USP) subvencionado pelo CNPq.

${ }^{1}$ Graduanda da EERP-USP. Bolsista de Iniciação Científica CNPq.

${ }^{2}$ Enfermeira. Professora Titular da EERP-USP.
}

Autorcorrespondente:

Emilia Campos de Carvalho

Av. Bandeirantes, 3900 - CEP 14040-902 - Ribeirão Preto - SP Recebido em: 11/08/07

E-mail: ecdcava@usp.br 


\section{INTRODUÇÃO}

$\mathrm{O}$ isolamento imposto pelo controle de infecções hospitalares e as características particulares do adoecer dos indivíduos internados, especialmente em uma unidade de Transplante de Medula Óssea (TMO), fazem com que os pacientes submetidos a este tratamento e seus familiares vivenciem situações de estresse ${ }^{(1-4)}$. A qualidade da assistência é um aspecto relevante para tais pacientes. Neste sentido, a comunicação terapêutica, entendida como a habilidade do profissional em ajudar uma pessoa a enfrentar uma situação temporária de estresse, a conviver com outras pessoas, a ajustar-se ao que não pode ser mudado e ainda a superar os bloqueios à auto-realização ${ }^{(5)}$, tornase imprescindível; possui, ainda, o potencial de favorecer o tratamento e o desenvolvimento dos pacientes, tornando-os ativos no processo do cuidado.

A comunicação ocorre quando as mensagens enviadas são claras, simples, transmitidas quando o receptor está apto para ouvir e quando há confiança, empatia e envolvimento emocional. Na comunicação terapêutica, o profissional assume a responsabilidade pela relação e a comunicação tem de ser planejada e com objetivos definidos, para ser útil para uma pessoa ou grupo. Uma das estratégias para se realizar uma comunicação terapêutica é o uso de técnicas de comunicação; deve-se ainda reconhecer os fenômenos não terapêuticos que ocorrem na comunicação ${ }^{(5-8)}$. A qualidade de um processo de comunicação sofre influência de vários fatores como condições do ambiente, condições emocionais, físicas, psicológicas e fisiológicas dos envolvidos, diferenças culturais, singularidade das pessoas, habilidade e competência do profissional, comunicação não-verbal adequada, entre outros. Portanto, não podemos afirmar que apenas a utilização destas técnicas terapêuticas irá tornar a comunicação terapêutica. As técnicas devem ser utilizadas de forma consciente, flexível, coerente, não repetitiva e, quando necessário, associadas às outras estratégias que facilitem o desenvolvimento de um relacionamento terapêutico ${ }^{(7)}$.

Diversos estudos abordam a relevância da comunicação entre enfermeiro e paciente ou seus familiares, em ambiente de $\mathrm{TMO}^{(2-4,9-10)}$, bem como outros $^{(11-12)}$ utilizam o referencial de Stefanelli, mas não se identificou estudos que usassem este referencial para pacientes de TMO.

Por outro lado, muitos achados na literatura evidenciam que a interação entre membros da equipe de enfermagem com outros profissionais e com pacientes é inadequada ${ }^{(13,14)}$, que o conhecimento sobre comunicação terapêutica e sobre as técnicas a serem utilizadas para alcançá-la pode ser melhorado e aprofundado por meio de educação permanente ${ }^{(5,14)}$. No processo ensino-aprendizado podem ser utilizadas estratégias como a exposição oral e a utilização do vídeo; estas podem auxiliar o trabalho educativo a aprofundar dimensões não percebidas e organizar o conhecimento em um projeto integral do ser humano ${ }^{(15)}$.

A comunicação, quando abordada ao longo da formação dos profissionais de Enfermagem, é tratada pelos cursos ou disciplinas com abordagens próprias e de forma inadequada, se considerarmos a complexidade das relações humanas ${ }^{(14)}$. Cabe lembrar que $46,7 \%$ das publicações nacionais de pesquisas sobre comunicação são aplicadas à área assistencial, enquanto a área educativa representa $25,2 \%$ destas $^{(13)}$.

Embora a comunicação seja aceita enquanto instrumento para a Enfermagem ${ }^{(13)}$, ocorre tanto o distanciamento do pessoal de serviço em relação ao conhecimento produzido por pesquisas, como sua não utilização.

Considerando que o conhecimento sobre habilidades comunicacionais contribui favoravelmente para mudanças no desempenho do profissional ${ }^{(14)}$, desenvolveu-se este estudo; partiu-se das hipóteses: que a equipe estudada empregava, tanto técnicas terapêuticas, em especial as de expressão, como não terapêuticas; que seus integrantes seriam capazes de identificar tais técnicas, mesmo sem exposição formal a elas, em situações similares às que cotidianamente empregam; que após a apresentação de um modelo teórico sobre o tema e o perfil de seus comportamentos terapêuticos e não terapêuticos, seriam capazes de alterar o padrão de conhecimento sobre os mesmos.

Para tanto, foram elaborados os seguintes objetivos:- verificar o conhecimento e a utilização da comunicação terapêutica pela equipe de enfermagem; verificar o efeito do uso de estratégia educativa sobre técnicas de comunicação terapêuticas e não terapêuticas, por meio da exposição oral, na mudança do padrão de respostas sobre o conhecimento das mesmas.

\section{MATERIAL E MÉTODOS}

Esta pesquisa foi desenvolvida em 4 fases; na primeira, com desenho descritivo, realizada de novembro de 2004 a janeiro de 2005 , foram observadas e registradas, de forma cursiva escrita, as comunicações verbais orais entre profissionais de enfermagem e pacientes durante a prestação dos cuidados, empregando-se dois observadores, com índice de concordância de $80 \%$. Os dados da presente pesquisa foram categorizados por uma das autoras, empregando-se as técnicas propostas ${ }^{(7-8)}$. A

Cogitare Enferm 2007 Jul/Set; 12(3):287-95 
categorização foi revista pela outra autora e o índice de concordância das análises foi de $98 \%(n=709)$, satisfatório para estudos dessa natureza.

Tendo em vista a hipótese prévia, de que a maior parte seria do grupo de expressão e que haveria a ocorrência de comunicação não terapêutica, programou-se a segunda fase do estudo para identificar o conhecimento de todas estas técnicas pelos profissionais. Realizada de julho a setembro de 2005 , consistiu em apresentar, inicialmente, um vídeo contendo três situações simuladas de interações entre profissional de enfermagem e paciente, contendo as técnicas de comunicação terapêuticas e não terapêuticas; os profissionais deveriam registrar em uma lista as técnicas que eles consideravam presentes ou não nas situações do vídeo.

Adotou-se a classificação das técnicas de comunicação terapêuticas nos grupos de Expressão, Clarificação e Validação, além de um grupo de técnicas de comunicação não terapêutica ${ }^{(7-8)}$.

A apresentação de cada situação durou cerca de cinco minutos. As cenas foram protagonizadas por uma enfermeira que atuou em uma unidade de TMO por cinco anos, tendo interpretado o papel de enfermeira; já o papel de paciente foi interpretado por uma das autoras desta pesquisa. O registro gravado ocorreu no Laboratório de Comunicação da Escola de Enfermagem de Ribeirão Preto - Universidade de São Paulo. Os diálogos e a ambientação retrataram situações observadas na primeira fase da pesquisa. A qualidade do vídeo e a pertinência da utilização das técnicas empregadas foram avaliadas por dois enfermeiros com experiência em pesquisa na área e conhecimento do referencial teórico, sendo consideradas adequadas à finalidade e aos objetivos do estudo.

A seguir, para cada uma das três situações, os profissionais de enfermagem deveriam registrar, em uma relação com todas as técnicas, quais consideraram presentes ou não no vídeo observado.

$\mathrm{Na}$ terceira fase, foi realizada a intervenção educativa, composta de uma apresentação oral sobre o conteúdo desse referencial teórico à equipe de enfermagem. Foi ministrada de acordo com a disponibilidade dos funcionários da unidade, nos seus turnos de trabalho, com duração de aproximadamente sessenta minutos. O conteúdo da exposição oral compreendeu os seguintes tópicos: conceito e finalidade da comunicação terapêutica; grupos e técnicas de comunicação terapêutica e não terapêutica; a contribuição da comunicação terapêutica no alcance dos objetivos estabelecidos; os possíveis efeitos do uso da comunicação não terapêutica, bem como as barreiras de comunicação. Este encontro permitiu que os profissionais tirassem suas dúvidas, discutissem as aplicações das técnicas de comunicação na prática, por meio de exemplos do seu cotidiano e conhecessem o perfil comunicacional observado pelo pesquisador, previamente. Solicitou-se que os profissionais respondessem a uma avaliação da exposição para conhecimento das pesquisadoras quanto à importância do tema e qualidade da exposição. Esta fase foi realizada no período de julho a outubro de 2006.

A seguir, na etapa final, quarta fase, o vídeo com as situações simuladas foi reapresentado e solicitada a identificação das técnicas presentes com o mesmo instrumento da fase dois. Foram sujeitos os profissionais que haviam participado das etapas anteriores da pesquisa, e que concordaram em participar dessa nova etapa.

Na comparação dos dados entre a segunda e quarta fases, caracterizando um estudo de desenho quase-experimental, foi empregado o teste de significância de diferença de duas proporções $(a ́=0,05)^{(16)}$ para comparação das proporções de acertos antes e depois da exposição teórica. O teste foi aplicado para os resultados de cada grupo de técnica de comunicação e para cada técnica.

Foram observados os preceitos éticos estabelecidos na Resolução 196 do MS, com submissão ao Comitê de Ética em Pesquisa da instituição.

Este estudo, descritivo foi desenvolvido em uma unidade de TMO de um hospital-escola, do interior paulista, que compreende sete leitos de internação, dos quais dois são isolamentos e uma Unidade de Hospital Dia. A unidade contava, na ocasião da coleta de dados, com equipe de enfermagem composta por dois enfermeiros, dois técnicos de enfermagem e oito auxiliares de enfermagem.

Para a coleta de dados foram empregados os seguintes instrumentos: Folha de registro dos diálogos, realizados de forma cursiva, contendo data, tipologia dos sujeitos e descrição das situações (empregado na primeira fase); Lista contendo as técnicas de comunicação terapêuticas e não terapêuticas ${ }^{(7-8)}$ para registro das opiniões dos profissionais sobre as categorias presentes no vídeo (empregado nas segunda e quarta fases); Vídeo contendo três simulações de interações profissionais de enfermagem-paciente; cada situação apresentava um conjunto de técnicas terapêuticas e não terapêuticas.

\section{RESULTADOS}

Foram sujeitos, nas duas primeiras fases, onze profissionais de enfermagem, pois, um deles estava 
ausente no período da coleta de dados. Destes, três eram do sexo masculino e oito do feminino; a faixa etária variou de 25 a 40 anos. Na terceira e na quarta fases, apenas nove dos sujeitos participaram, dos quais dois eram do sexo masculino. Os outros dois não participaram por demissão e férias. Foram também sujeitos, na primeira fase, vinte pacientes, sendo nove do sexo masculino, com dezoito anos ou mais e em condições de comunicar-se verbalmente.

Os diálogos entre profissionais de enfermagem e pacientes contemplam o emprego dos diferentes grupos de técnicas de comunicação, totalizando 709 $(100 \%)$ técnicas, das quais $612(86.3 \%)$ são terapêuticas e 97 (13,6\%) não terapêuticas.

Conforme previsto na hipótese inicial, dentre as terapêuticas, as técnicas que correspondem ao grupo de expressão foram as mais utilizadas $(80,8 \%)$; os grupos de clarificação $(4,3 \%)$ e de validação $(1,1 \%)$ foram pouco evidentes. Este não é um padrão usual ou específico apenas desta amostra. Resultados similares foram obtidos com alunos interagindo com pacientes com transtornos psiquiátricos ${ }^{(17)}$, como também por docentes e pelo pessoal assistencial durante terapia endovenosa, em estudo realizado no México ${ }^{(12)}$.

Embora seja o grupo mais empregado, apenas algumas das técnicas de expressão foram observadas em elevada freqüência. Elas foram: fazer perguntas e usar frases descritivas (40,3\% cada); as técnicas colocar em foco a idéia principal e dizer não, não foram observadas. As outras apresentaram freqüências iguais ou inferiores a $1 \%$, sendo pouco evidentes.

As técnicas fazer pergunta e usar frases descritivas correspondem a $75,4 \%$ do total de todas as técnicas de comunicação terapêutica $(n=612)$ utilizadas pelos profissionais. Este padrão de comunicação mostra que os profissionais de enfermagem não estão desenvolvendo um relacionamento terapêutico pleno. A maior parte das perguntas e das frases descritivas utilizadas pelos profissionais do TMO referia-se à alimentação, medicamentos, procedimentos que foram ou que seriam realizados e situação fisiológica do paciente. Estas perguntas não davam continuidade ao diálogo.

A utilização de forma apropriada das diversas técnicas de comunicação terapêutica do grupo de expressão poderia encorajar maiores e melhores interações, porque demonstrariam aceitação e respeito. Elas estimulam ou facilitam a descrição de experiências, sentimentos e pensamentos dos pacientes; constitui-se na primeira etapa do processo de comunicação terapêutica e torna possível que o enfermeiro conheça o paciente para depois clarificar e validar os conteúdos das interações, podendo identificar problemas e intervir.

No presente estudo, as técnicas de comunicação terapêutica do grupo de clarificação mais utilizadas pelos sujeitos foram descrever os eventos em seqüência lógica (52,9\%) e estimular comparações $(23,5 \%)$. O emprego destas técnicas pode indicar uma comunicação baseada em transferência de informações da equipe para o paciente (ou vice-versa), sem maior envolvimento, pois foram aplicadas em grande parte abordando os temas relacionados a medicamento, procedimentos, rotina da unidade e condições fisiológicas do paciente. Contudo, tais técnicas possibilitam que as mensagens tornemse claras e compreensíveis ao paciente, reduzindo ansiedade e dúvidas que acarretam estresse ${ }^{(7)}$.

Os dados relativos ao grupo de validação mostram que estas técnicas, no computo geral, foram observadas em reduzida freqüência $(1,1 \%)$. Dentre estas, houve predomínio do uso da técnica repetir a mensagem do paciente $(87,5 \%)$. Por outro lado, em apenas uma situação houve resumir o conteúdo da interação; já a técnica pedir ao paciente para repetir $o$ que foi dito, não foi utilizada. $\mathrm{O}$ grupo de validação serve para certificar que o conteúdo da interação foi corretamente compreendido pelos interlocutores. Se os profissionais de enfermagem utilizassem tais técnicas com maior freqüência e nos momentos ideais (paciente apto, com tempo e ambiente adequados para interagir com os profissionais) poderiam reduzir as situações de não entendimento sobre a rotina da unidade, tratamento ou procedimentos, favorecendo o caminho para o autocuidado e o desenvolvimento de uma comunicação terapêutica.

Para ocorrer um relacionamento terapêutico com os pacientes, faz-se necessário a utilização dos três grupos de técnicas de comunicação descritos. Porém, das interações observadas, $13,6 \%$ retratam o uso de técnicas de comunicação não terapêuticas. Dentre estas, induzir respostas (17,5\%), falsa tranqüilização $\quad(16,4 \%), \quad$ comunicar-se unidirecionalmente $(15,4 \%)$, mudar de assunto subitamente $(13,4 \%)$ e julgar o comportamento $(10,3 \%)$ foram as técnicas de comunicações não terapêuticas mais freqüentes; no entanto, todas as técnicas de comunicação não terapêuticas apareceram nos registros, com menores freqüências.

No processo de comunicação interpessoal, os envolvidos se influenciam ou se afetam recíproca ou mutuamente. Sendo assim, a ocorrência da comunicação 
não terapêutica traz prejuízos para quem a usa e para o outro envolvido no processo de comunicação.

Frases imperativas, perguntas retóricas e o uso de diminutivos foram observados neste estudo. Estas situações podem negar ao paciente o direito de ser uma pessoa com seus próprios modos de agir, pensar e sentir, podendo ocorrer ainda um apagamento da individualidade do enfermeiro, em que este passa a ser tratado como um coletivo pelos pacientes ${ }^{(17)}$.

A competência em comunicação deve ser associada ao desempenho da função técnica, para que o paciente tenha um cuidado de alta qualidade científica e humanitária. Ao enfermeiro cabe tornar sua comunicação o mais eficiente possível, pois cada momento é único e muitas vezes decisivo para a tomada de decisão em relação às condutas a serem seguidas ${ }^{(18)}$.

Considerando que ter compreensão da complexidade comunicacional que envolve o processo de enfermagem favorece a valorização e a busca de habilidades para cumprir melhor os objetivos de sua aplicação, seja em que contexto for, estão apresentados os resultados da identificação das categorias de comunicação, pelos sujeitos, nos vídeos, nas etapas pré e pós-intervenção.

Das 1056 respostas esperadas, na fase préintervenção, 664 (62,87\%) foram corretas; as técnicas do grupo de expressão foram as mais utilizadas pelos profissionais de enfermagem; no entanto, as técnicas identificadas corretamente, com maiores freqüências pela equipe, foram as do grupo de validação. A linha de base do conhecimento dessas técnicas, nessa fase, ficou assim estabelecida:

Tabela 1 - Freqüência das técnicas de comunicação identificadas pelos profissionais de enfermagem em situações apresentadas em vídeo, Ribeirão Preto, 2006

\begin{tabular}{lccc}
\hline $\begin{array}{l}\text { Grupo de } \\
\text { técnicas }\end{array}$ & $\begin{array}{c}\mathbf{N}^{\mathbf{0}} \text { total de } \\
\text { respostas }\end{array}$ & $\begin{array}{c}\mathbf{N}^{\mathbf{0}} \text { de } \\
\text { acertos }\end{array}$ & $\begin{array}{c}\text { \% de } \\
\text { acertos }\end{array}$ \\
\hline Expressão & 495 & 297 & 60,0 \\
Clarificação & 132 & 89 & 67,4 \\
Validação & 99 & 79 & 79,7 \\
Não Terapêuticas & 330 & 199 & 60,3 \\
\hline
\end{tabular}

A utilização das técnicas de um determinado grupo parece que não se baseia em ser capaz de identificar ou não as mesmas. Embora não as empreguem, foram identificadas corretamente em, pelo menos, $60 \%$ das situações. Cabe destacar que as técnicas do grupo de validação possuem denominações facilmente associáveis às situações apresentadas em vídeo, sendo as identificadas com maior freqüência pelos profissionais. É desejável que os profissionais saibam reconhecer todas as técnicas descritas, de todos os grupos, incluindo as não terapêuticas, para que haja a possibilidade de reflexões sobre sua prática e, posteriormente, mudanças no seu perfil comunicacional.

O profissional de enfermagem, para alcançar os objetivos inerentes à sua função, deve estabelecer, com o paciente uma relação que desperte confiança, em que este possa falar de si livremente, possibilitando ao profissional detectar quais são suas necessidades, sendo esta a primeira etapa da assistência ${ }^{(19)}$. As técnicas do grupo de expressão podem auxiliar o profissional neste processo, daí a expectativa de que empreguem e reconheçam tais técnicas. As diferenças na identificação de tais técnicas, nos dois momentos, constam da Tabela 2.

Tabela 2 - Diferença de proporção das técnicas de comunicação terapêutica do grupo de expressão corretamente identificadas pelos profissionais de enfermagem, Ribeirão Preto, 2006

\begin{tabular}{lcc}
\hline Técnicas de comunicação & $\begin{array}{c}\text { Diferença } \\
\text { das } \\
\text { proporções }\end{array}$ & $\begin{array}{c}\text { Teste de } \\
\text { significância } \\
\text { (Z) }\end{array}$ \\
\hline Ouvir reflexivamente & 9,7 & 0,097 \\
Verbalizar dúvidas & 14,8 & 1,1644 \\
Estimular a expressão de & & \\
sentimentos subjacentes & 23,6 & 1,8112 \\
Usar terapeuticamente o humor & 6,1 & $-1,2973$ \\
Verbalizar aceitação & 6,7 & 0,5723 \\
Verbalizar interesse & 11,1 & 0,8795 \\
Usar frases com sentido aberto & 38,4 & $-2,99$ \\
ou reticentes & & \\
Repetir comentários ou últimas & 37,7 & $-2,9134$ \\
palavras ditas pelo paciente & 1,4 & 0,2160 \\
Fazer pergunta & 7,1 & $-0,5478$ \\
Devolver a pergunta feita & 0,7 & 0,056 \\
Usar frases descritivas & & \\
Permitir ao paciente que escolha & 15,8 & $-1,2519$ \\
o assunto & 7,4 & $-0,6223$ \\
Usar terapeuticamente o silêncio & 0 & 0 \\
Colocar em foco a idéia principal & 7,4 & $-0,6223$ \\
Dizer não & & \\
\hline
\end{tabular}

No grupo de expressão, sete técnicas apresentaram mudanças, ampliando o índice de acertos nos resultados; contudo tais mudanças não foram significantes para as técnicas Usar 
terapeuticamente o humor, Devolver a pergunta feita, Permitir ao paciente que escolha o assunto, Usar terapeuticamente o silêncio e Dizer não. Somente as técnicas Usar frases com sentido aberto ou reticentes e Repetir comentários ou últimas palavras ditas pelo paciente apresentaram alterações positivas estatisticamente significativas $(\mathrm{P}<-1,64)$. Estas técnicas foram as que obtiveram menores índices de acerto na fase 2 desse estudo.

Outras sete técnicas apresentaram quedas nos índices de acertos; contudo tais mudanças não foram estatisticamente significantes para seis delas: Ouvir reflexivamente, Verbalizar dúvidas, Verbalizar aceitação, Verbalizar interesse, Fazer pergunta e Usar frases descritivas. Já a técnica Estimular a expressão de sentimentos subjacentes apresentou uma queda estatisticamente significativa $(p>1,64)$ em seu índice de acerto, sendo que na fase inicial apresentou escore de $60,6 \%$ de acerto. O conhecimento destas estratégias é relevante, pois são informações a serem transmitidas e seguidas pelos pacientes.

Observações podem ser suscetíveis a erros; os profissionais podem não ter realizado uma observação de forma sistemática, sabendo o que procuravam e mantendo a concentração ${ }^{(12)}$. Os resultados da observação do vídeo dependeram do conhecimento destes profissionais e da sua percepção dos fenômenos de difícil identificação, na medida em que cada situação é vista a partir de diferentes prismas.

Por outro lado, há que se considerar, para todos os grupos de técnicas, que a exposição do vídeo, acompanhada da relação das técnicas nele contidas, também é um estímulo à aprendizagem, e o nível inicial de reconhecimento de tais técnicas foi de $60 \%$. O que pode ter gerado maior dificuldade para ocorrer mudança.

Neste trabalho, o fenômeno é audiovisual e a observação inclui o treinamento de capacidades que são obtidas na formação educacional para o trabalho em enfermagem como, por exemplo, a comunicação não-verbal. A interferência dos valores de cada um, que está implícita nas percepções das situações observadas, também é outro fator a ser considerado.

As técnicas do grupo de clarificação possibilitam que o profissional possa compreender a mensagem do paciente e ser compreendido.

Tabela 3 - Diferença de proporção das técnicas de comunicação terapêuticas dos grupos clarificação e validação corretamente identificadas pelos profissionais de enfermagem, Ribeirão Preto, 2006

\begin{tabular}{llcc}
\hline Grupo & Técnicas de comunicação & $\begin{array}{c}\text { Diferença das } \\
\text { proporçóes }\end{array}$ & $\begin{array}{c}\text { Teste de } \\
\text { significância (Z) }\end{array}$ \\
\hline Clarificação & Estimular comparações & 10,4 & 0,8407 \\
& Solicitar ao paciente que esclareça termos incomuns & 25,9 & 2,0077 \\
& Solicitar ao paciente que precise o agente de ação & 16,5 & $-1,333$ \\
& Descrever os eventos em sequiência lógica & 2 & $-0,1821$ \\
Validação & Repetir a mensagem do paciente & 25,2 & 1,9702 \\
& Pedir ao paciente para repetir o que foi dito & 5,1 & 0,7112 \\
& Resumir o conteúdo da interação & 9,4 & $-0,9064$ \\
\hline
\end{tabular}

Duas técnicas deste grupo apresentaram queda no índice de acertos na segunda fase; contudo, somente Solicitar ao paciente que esclareça termos incomuns apresentou mudança estatisticamente significativa, $(\mathrm{P}>1,64)$, observando-se melhor desempenho dos profissionais na primeira etapa da pesquisa (que correspondeu a $66,6 \%$ de acertos). A relevância do uso destas técnicas alicerça-se no fato de que quando o paciente se sente incompreendido, suas insatisfações, dúvidas e medos aumentam, podendo levar ao inconformismo em relação ao tratamento e até mesmo à não colaboração ${ }^{(12)}$. A outra técnica, Estimular comparações, foi uma das que tiveram maior índice de acerto $(69,6 \%)$ na fase pré-intervenção; ela leva o paciente a refletir sobre experiências anteriores semelhantes para tentar encontrar soluções para seu atual problema, reduzindo a ansiedade. Descrever os eventos em seqüência lógica auxilia na educação do paciente e de seus familiares, intervenção relevante no cuidado ao paciente ${ }^{(20)}$.

As técnicas Solicitar ao paciente que precise o agente de ação e Descrever os eventos em seqüencia lógica mudaram de forma positiva, observando-se discreta elevação no índice de acerto, na segunda fase. Foram observados $57,5 \%$ e $75,7 \%$ de acertos na fase inicial respectivamente. Toda 
comunicação implica em um compromisso que define a relação, assim, uma informação não é somente transmitida, ela impõe um comportamento definindo relações diferentes que raramente são estabelecidas de modo deliberado e com plena consciência ${ }^{(12)}$. Este fato torna essencial a clarificação das mensagens emitidas por todos envolvidos no processo de comunicação.

A complexidade do ensino de comunicação e as dificuldades de se obter mudanças comportamentais têm sido apresentadas na literatura. Um dos aspectos dificultadores nessa mudança é o uso restrito de técnicas que propiciam a compreensão e autoexploração (técnicas dos grupos de clarificação e validação) e, portanto, que promovem mudanças, bem como o fato de se atribuir à comunicação relevância secundária com relação às patologias e procedimentos. Cabe destacar, ainda, que a ansiedade pode dificultar o desenvolvimento de habilidades ${ }^{(14)}$.

O grupo de validação permite aos profissionais e ao paciente confirmarem o conteúdo da interação, gerando a possibilidade de reflexão sobre suas condutas e correções, quando necessário. É, portanto, desejável o emprego freqüente destas técnicas assim como o seu reconhecimento.

As técnicas do grupo de validação foram as menos utilizadas pelos profissionais de enfermagem do setor em estudo, quando observado seu padrão de comunicação ${ }^{(2)}$; contudo, foram as que apresentaram maior índice de acertos na associação das mesmas com a exposição do vídeo: A técnica Repetir a mensagem do paciente foi a mais utilizada dentre elas, embora tenha sido a menos identificada pelos profissionais de enfermagem nas situações simuladas nas duas etapas, observando-se a queda, estatisticamente significativa ( $\mathrm{p}>1,64)$, no índice de acertos. A técnica Resumir o conteúdo da interação obteve aumento do índice de acerto na segunda etapa, e houve redução desse índice para a técnica Pedir ao paciente para repetir o que foi dito.

Cabe lembrar que, durante a intervenção educativa, os profissionais referiram ter dificuldades para aplicar as técnicas de comunicação terapêuticas, uma vez que muitos pacientes apresentavam-se ansiosos, agressivos, deprimidos ou simplesmente não desejavam falar, ou ainda, por outro lado, devido ao excesso de atividades da Enfermagem. Em uma interação há coresponsabilidade e os profissionais da saúde possuem conhecimento para iniciar o contato com o paciente, independentemente das dificuldades, não devendo colocar o paciente como responsável pela utilização ou não de uma comunicação mais adequada ${ }^{(12)}$.

$\mathrm{O}$ resultado desta pesquisa aponta que os profissionais têm dificuldade de refletir sobre o seu desempenho na comunicação com o paciente. Esse aspecto corrobora o fato de que algumas escolas apresentam esse conteúdo, pontualmente, na formação de seus alunos, bem como centram suas preocupações na execução de procedimentos e não na reflexão sobre a situação que a eles se relaciona ${ }^{(21)}$.

Outro aspecto do reconhecimento do padrão comunicacional pelos sujeitos é que o relacionamento enfermeiro-paciente é influenciado pelo dia-a-dia no hospital e por muitos fatores que direcionam a assistência para o plano biomédico e procedimental. Estes são realizados, muitas vezes, rotineiramente e por um relacionamento unidirecional, apenas de instruções ou informações ${ }^{(12)}$. Vários aspectos da atividade profissional (contato com pessoas doentes, problemas psicossociais, responsabilidades delegadas, entre outros) deixam os profissionais de enfermagem vulneráveis aos efeitos negativos do trabalho, gerando um estresse ocupacional. Estes aspectos devem ser considerados, em especial quanto ao uso de técnicas não terapêuticas, não desejáveis durante a comunicação com paciente.

Tabela 4 - Diferença de proporção das técnicas de comunicação não terapêuticas identificadas corretamente pelos profissionais de enfermagem, Ribeirão Preto, 2006

\begin{tabular}{lcc}
\hline Técnicas de comunicação & $\begin{array}{c}\text { Diferença } \\
\text { das } \\
\text { proporções }\end{array}$ & $\begin{array}{c}\text { Teste de } \\
\text { significância } \\
(\mathbf{Z})\end{array}$ \\
\hline Comunicar-se & 9,4 & $-0,7264$ \\
unidirecionalmente & 21,5 & $-1,8728$ \\
Pôr o paciente à prova & 2,7 & $-0,3064$ \\
Induzir respostas & 9,1 & $-0,7216$ \\
Falsa tranqüilização & & \\
Usar jargões técnicos ou & 8,4 & 0,7479 \\
linguagem científica & 12,1 & 1,0540 \\
Manter-se na defensiva & 11,4 & $-0,8864$ \\
Julgar o comportamento & & \\
Mudar de assunto & 1,4 & 0,2160 \\
subitamente & 6,4 & 0,6911 \\
Não saber ouvir & 15,5 & $-1,2062$ \\
Dar conselho & &
\end{tabular}

Nota-se que quatro técnicas (Usar jargões técnicos ou linguagem científica; Manter-se na 
defensiva; Mudar de assunto subitamente e Não saber ouvir) apresentaram redução do índice de acertos na segunda etapa da pesquisa; na fase inicial obtiveram elevados índices de acertos, $>78,7 \%$. As outras seis técnicas não terapêuticas foram identificadas corretamente, com maiores escores. Dentre estas, apenas Pôr o paciente à prova apresentou diferença estatisticamente significativa $(\mathrm{p}<-1,64)$, no entanto, os resultados não foram satisfatórios, pois a maioria não apresentou mudanças no padrão de respostas.

De forma global, os resultados categorizados por grupo, nas situações antes e após a exposição oral, não apresentam diferenças estatisticamente significativas, embora se observem mudanças no padrão de respostas com aumento para os grupos de expressão e não terapêuticas e redução para os grupos de clarificação e validação.

Tabela 5 - Diferença de proporção das técnicas de comunicação corretamente identificadas pelos profissionais de enfermagem, Ribeirão Preto, 2006

\begin{tabular}{lcc}
\hline Grupo de técnicas & $\begin{array}{c}\text { Diferença das } \\
\text { proporções }\end{array}$ & $\begin{array}{c}\text { Teste de } \\
\text { significância (Z) }\end{array}$ \\
\hline Expressão & 3,4 & $-1,0029$ \\
Clarificação & 4,5 & 0,7299 \\
Validação & 6,9 & 1,088 \\
Não Terapêuticas & 4,1 & $-1,03$ \\
\hline
\end{tabular}

A avaliação feita pelos sujeitos desta experiência de ensino foi positiva para $81,8 \%$ dos participantes, que consideraram o conteúdo da exposição útil; $63,6 \%$ certamente recomendariam a aula a um colega e $63,6 \%$ gostariam de assistir outras aulas ou cursos que aprofundassem o tema abordado e ainda, consideraram pequeno o tempo de exposição. $\mathrm{Na}$ avaliação, surgiram sugestões para a inclusão do ensino de comunicação terapêutica durante a formação do auxiliar e do técnico de enfermagem.

Identifica-se, contudo, a necessidade de rever a estratégia utilizada, uma vez que não foi alterado o comportamento de base, embora se destaque que a identificação correta das técnicas empregadas no vídeo, na primeira fase, já apresentava índice de desempenho superior a $60 \%$. A estratégia utilizada para proporcionar o conhecimento das técnicas - a exposição oral - pode não ter sido efetiva. Ainda, a relação tempo e quantidade de exposições (uma apresentação de aproximadamente 60 minutos) pode ter interferido no comportamento dos profissionais. Sugere-se conhecer, futuramente, a opinião dos profissionais quanto à qualidade do padrão comunicacional que utilizam, pois podem considerar que não há necessidade de modificá-lo.

Os processos de relações interpessoais, para serem produtivos e resultando em processo de ajuda, colaboração e amadurecimento psicológico, necessitam que um dos elementos tenha conhecimento das implicações da relação e consciência da sua parcela de responsabilidade quanto aos resultados obtidos em um relacionamento terapêutico ${ }^{(9)}$.

\section{CONSIDERAÇÕES FINAIS}

O conhecimento e a utilização da comunicação terapêutica pela equipe de enfermagem observados podem ser melhorados e aprofundados, considerando as técnicas mais utilizadas (fazer pergunta e usar frases descritivas) e as mais identificadas pelos profissionais de enfermagem (fazer pergunta, pedir ao paciente para repetir o que foi dito, mudar de assunto subitamente e usar terapeuticamente o humor).

$\mathrm{O}$ efeito da intervenção (exposição oral) na identificação das técnicas de comunicação terapêuticas e não terapêuticas, em simulações da interação entre enfermeiro e paciente, mostrou-se estatisticamente significante para três das técnicas do grupo de expressão, para uma dentre as técnicas do grupo de clarificação e uma do grupo de validação. Contudo, examinando-se os grupos em sua totalidade, não se obteve mudanças estatisticamente significativas, embora mudanças consideradas desejadas fossem observadas, como o aumento do grupo de expressão. Ocorreu redução para os outros dois grupos, o que não era esperado.

Quanto às variações dos índices de acertos para as técnicas não terapêuticas de comunicação, foram identificadas mudanças estatisticamente significantes apenas para uma das dez técnicas contidas nesse grupo. Seis delas tiveram ampliação nos índices de acerto na segunda fase do estudo, o que era desejável. De forma global, não houve mudança significativa para o conjunto das técnicas desta categoria, embora tenham sido mais identificadas após a intervenção.

Os profissionais trouxeram interesses em incluir e conhecer abordagens da comunicação na relação com a equipe de trabalho, na vida pessoal e com os acompanhantes dos pacientes, indicando que outras necessidades estavam presentes no momento da pesquisa.

Considera-se que outros métodos de formação são necessários para esta equipe e que despertar para formas mais terapêuticas de comunicação, reduzindo 
a utilização da comunicação não terapêutica, deve ser meta dos profissionais de enfermagem para que ocorra transformação no processo de trabalho.

O desenvolvimento de uma comunicação terapêutica entre equipe de enfermagem e pacientes pode propiciar um cuidado integral e de maior qualidade, principalmente em ambientes como a unidade de TMO, onde as situações de estresse são constantes para os envolvidos no processo de comunicação.

\section{REFERÊNCIAS}

1 Contel JOB. Aspectos psicológicos e psiquiátricos do transplante de medula óssea. Medicina Ribeirão Preto. $2000 \mathrm{Jul} / \mathrm{Set} ; 33: 294-311$.

2 Krasuska ME, Stanislawek A. Communication with patients and their families, who undergo bone marrow transplantation. Ann Univ Mariae Curie Sklodowska. 2003;58(2):168-73.

3 Stetz KM, McDonald JC, Compton K. Needs and experiences of family caregivers during marrow transplantation. Oncol Nurs Forum. 1996 Oct; 23(9):1422-7.

4 Phipps S, Dunavant M, Lensing S, Raí SN. Psychosocial predictors in parents of children undergoing stell cell or bone marrow transplantation. J Ped Psychol. 2000 Mar;30(2):139-53.

5 Stefanelli MC. Introdução à comunicação terapêutica. In: Stefanelli MC, Carvalho EC. A comunicação nos diferentes contextos da enfermagem. Barueri: Manole; 2005. p.62-72.

6 Stefanelli MC. Comunicação com paciente: teoria e ensino, $2^{\mathrm{a}}$ ed. São Paulo: Robe; 1993.

7 Stefanelli MC. Estratégias de comunicação terapêutica. In: Stefanelli MC, Carvalho EC. A comunicação nos diferentes contextos da enfermagem. Barueri: Manole; 2005.p.73-104.

8 Stefanelli MC. Comunicação não terapêutica e barreiras à comunicação terapêutica. In: Stefanelli MC, Carvalho EC. A comunicação nos diferentes contextos da enfermagem. Barueri: Manole; 2005. p.105-17.

9 Shulmeister L, Quiett K, Mayer K. Quality of life, quality of care and patient satisfaction: perceptions of patients undergoing outpatient autologous stem cell transplantation. Oncol Nurs Forum. 2005 Jan;32 (1):57-67.

10 Carvalho EC, Gonçalves PG, Bontempo APM, Soler VM.
Interpersonal needs expressed by patients during bone marrow transplantation. Cancer Nursing. 2000 Dec; 23(6):462-7.

11 Sadala MLA, Stefanelli MC. Avaliação do ensino de relacionamento enfermeira-paciente. Rev Latino-am Enferm. 1996 Abr; 4(especial):139-52.

12 Landeros LM, Carvalho EC. La comunicación terapéutica durante instalación de venoclisis: uso de la simulación filmada. Rev Latino-am Enferm. 2006 Out;14(5):658-65.

13 Carvalho EC. O conhecimento sobre comunicação em enfermagem na literatura internacional. Rev Paul Enferm. 1991 Set/Dez;10(3):108-14.

14 Carvalho EC, Bachion MM, Almeida LCP, Medeiros RN. O ensino de comunicação em enfermagem: um desafio. Rev Latino-am Enferm. 1997 Jul;5(3):27-34.

15 Moran JM. Interferências dos meios de comunicação no nosso conhecimento. Rev INTERCOM - Revista Brasileira de Comunicação 1994 Jul/Dez;17(2):38-49.

16 Pagano M, Gauvreau K. Princípios de Bioestatística. São Paulo: Pioneira Thomson Learning; 2004.

17 Tfouni LV. O discurso na instituição hospitalar. Anais do $1^{\circ}$ SIBRACEn; 1988 502-12; Ribeirão Preto-SP; 1988.

18 Carvalho EC, Bachion MM. Comunicação e o processo de enfermagem. In: Stefanelli, MC Carvalho EC. A comunicação nos diferentes contextos da enfermagem. Barueri: Manole; 2005. p.138-56.

19 Carneiro A. Teoria de Roger e suas aplicações no campo da enfermagem. Rev Gaúcha Enferm. 1986 Jul;7(2):265-74.

20 Yoon SJ, Conway J, McMilan M. An exploration of the concept of patient education: implications for the development of educational programmes for relapsed post bone marrow transplantation patients and their families in Korea. Int J Nurs Pract. 2006 Jun;12(3):129-35.

21 Carvalho EC, Pelá NTR. O ensino de comunicação a enfermeiros através do Sistema Personalizado de Instrução. Anais do $2^{\circ}$ Simpósio Brasileiro de Comunicação em Enfermagem. Ribeirão Preto (SP): Escola de Enfermagem de Ribeirão Preto; 1990. p. 566 - 80. 